\title{
Robotic cardiac surgery in Brazil
}

\section{Robinson Poffo, Alisson P. Toschi, Renato B. Pope, Paola K. Montanhesi, Ricardo S. Santos, Alexandre Teruya, Dina M. Hatanaka, Gabriel F. Rusca, Claudio H. Fischer, Marcelo C. Vieira, Marcia R. Makdisse}

Centro de Cirurgia Cardíaca Minimamente Invasiva e Robótica, Hospital Israelita Albert Einstein, São Paulo, SP, Brazil

Correspondence to: Robinson Poffo. Av. Albert Einstein, 672/701, Bloco A-1, 4 andar, sala 421, CEP 05652-901, Sao Paulo, SP, Brazil.

Email: robinson.poffo@einstein.br.

Background: Brazil, the largest country and economy in South America, is a major driving force behind the development of new medical technologies in the region. Robotic cardiac surgery (RCS) has been evolving rapidly since 2010 , when the first surgery using the $\mathrm{DaVinci}{ }^{\circledR}$ robotic system was performed in Latin America. The aim of this article is to evaluate short and mid-term results in patients undergoing robotic cardiac surgery in Brazil.

Methods: From March 2010 to December 2015, 39 consecutive patients underwent robotic cardiac surgery. Twenty-seven patients were male (69.2\%), with the mean age of $51.3 \pm 17.9$ years. Participants had a mean ejection fraction of $62 \pm 5 \%$. The procedures included in this study were mitral valve surgery, surgical treatment of atrial fibrillation, atrial septal defect closure, resection of intra-cardiac tumors, totally endoscopic coronary artery bypass and pericardiectomy.

Results: The mean time spent on cardiopulmonary bypass (CPB) during RCS was $154.9 \pm 94.2$ minutes and the mean aortic cross-clamp time was $114.48 \pm 75.66$ minutes. Thirty-two patients $(82 \%)$ were extubated in the operating room immediately after surgery. The median intensive care unit (ICU) length of stay was 1 day (ranging from 0 to 25) and the median hospital length of stay was 5 days (ranging from 3 to 25). For each type of procedure, endpoints were individually reported. There were no conversions to sternotomy and no intra-operative complications. Patient follow-up was complete in $100 \%$ of the participants, with two early deaths unrelated to the procedures and no re-operations at mid-term.

Conclusions: Despite the heterogeneity of this series, RCS appears to be feasible, safe and effective when used for the correction of various intra- and extra-cardiac pathologies. Adopting the robotic system has been a challenge in Brazil, where its limited clinical application may be related to the lack of specific training and the high cost of technology.

Keywords: Minimally invasive surgery; robotic surgery; valve surgery; Coronary artery bypass; atrial septal defect

Submitted Mar 11, 2016. Accepted for publication Oct 30, 2016.

doi: 10.21037/acs.2017.01.01

View this article at: http://dx.doi.org/10.21037/acs.2017.01.01

\section{Introduction}

Robotic-assisted totally endoscopic cardiac surgery has been performed for more than fifteen years in Europe and United States of America (1-3). Since the first procedures, there have been extensive discussions regarding its safety, viability and clinical outcomes (4). A growing number of large clinical centers have become involved with use of this new modality, with centers showing particular interest in the potential for reduced surgical trauma. The proposed benefits of reduced tissue manipulation and trauma include shorter length of hospital stay, earlier return to social and professional activities and greater patient satisfaction when compared with conventional procedures (4).

Recent changes in generalized access to medical information through the internet have increased patient desire for less invasive procedures (5). Increasing numbers of patients are pursuing new technologies and procedures that promise to improve their quality of life, minimize postoperative pain and accelerate recovery following 


\section{Robotic surgery in Hospital Israelita Albert Einstein according to surgical specialties from 2008 to 2015}

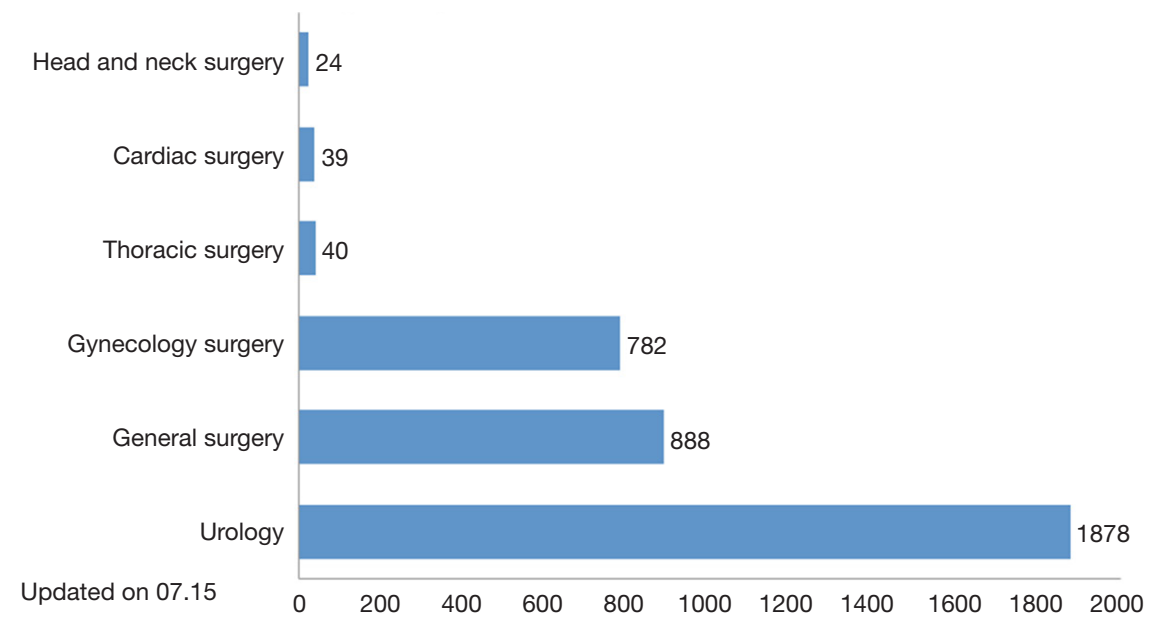

Figure 1 Robotic Surgery in Hospital Israelita Albert Einstein according to surgical specialties from 2008 to 2015.

surgical treatment. Medical institutions worldwide must continue to maintain a high level of excellence and to follow international guidelines of safety and quality of assistance (6).

In 2008, three private hospitals in the state of Sao Paulo incorporated robotic technology into their surgical armamentarium; however, only Hospital Israelita Albert Einstein implemented programs for all surgical specialties, including urology, gynecology, gastrointestinal, head and neck, thoracic and cardiac surgery. From 2008 to 2015, 3,651 patients underwent robotic surgery at Hospital Israelita Albert Einstein (Figure 1), corresponding to the majority of cases operated in the whole country. In 2010, the first robotic cardiac surgery in Latin America using the $\mathrm{DaVinci}^{\circledR}$ robotic system (Intuitive Surgical, Inc., Sunnyvale, CA, USA) was performed and, since then, 39 surgeries have been performed. Hospital Israelita Albert Einstein remains the only hospital robotic technology to use for cardiac surgery in Brazil.

This article aims to report the results of the first series of cardiac robotic surgeries performed in Brazil. The objectives of this study are to evaluate the short- and mid-term outcomes of patients undergoing robotic cardiac surgery in a general, tertiary-care, and philanthropic hospital.

\section{Methods}

This prospective study includes 39 consecutive patients who underwent robotic-assisted cardiac surgery using the DaVinci ${ }^{\circledR}$ robotic system (Intuitive Surgical, Inc.,
Sunnyvale, CA, USA), composed of a set of four robotic arms, an image capture and recording system and a console through which the surgeon controls the robot's movements.

All participants gave written informed consent. Participants were provided with information on the surgical possibilities regarding the performance of the procedure and chose the robotic-assisted approach. The study was approved by the Hospital Israelita Albert Einstein ethics committee under the number CEP/Einstein 11/1501, entitled "Einstein Registry of Robotic-assisted and Minimally Invasive Cardiovascular Surgery".

The procedures were performed between March 2010 and December 2015. Inclusion criteria followed the indications for conventional surgical correction of cardiac pathologies, whether acquired or congenital. Exclusion criteria for the performance of the robotic-assisted procedures included patients with thoracic deformities; severe thoracic trauma; anatomical or pathological abnormalities of the peripheral vascular system; moderate or severe aortic regurgitation; ejection fraction $<40 \%$; highly calcified coronary arteries, vessels with a diameter $<1.5 \mathrm{~mm}$ or multi-vessel disease.

Patients who had mitral valve disease were screened using trans-esophageal echocardiographym (TEE). Surgical correction was indicated following the American College of Cardiology (ACC) and the American Heart Association (AHA) guidelines for the management of patients with valve disease (7). Patient follow-up was performed in an outpatient setting, consisting of regular clinical evaluation, 

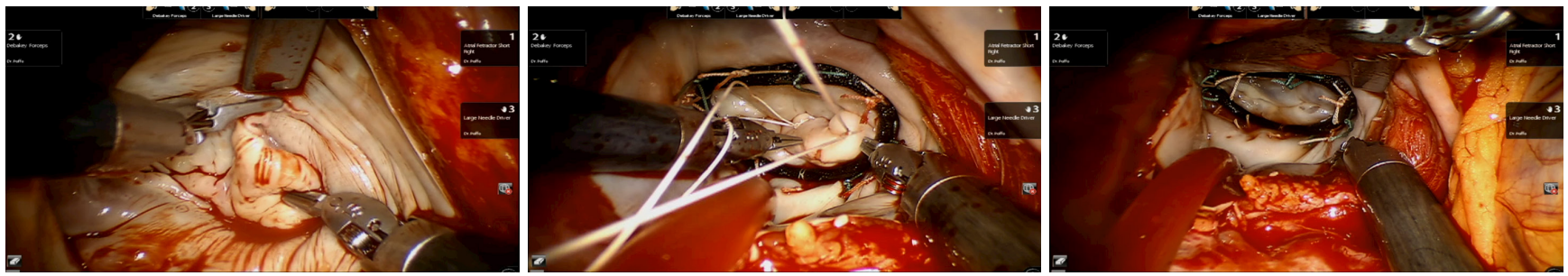

Figure 2 Mitral valve regurgitation repair (P2 prolapse) due to a ruptured chordae using polytetrafluoroethylene neo chords and a complete semi-rigid ring.

New York Heart Association (NYHA) classification and echocardiographic findings.

\section{Surgical technique}

\section{Mitral valve operations}

Robotic mitral valve repair or replacement was performed using a technique previously described by Chitwood et al. (8). Patients were intubated using a Robert Shaw endotracheal tube for selective pulmonary ventilation and positioned with the right hemi-thorax elevated at $20^{\circ}$. Disposable paddles for external cardiac defibrillation were placed in the right scapular and anterolateral region of the left hemi-thorax. A nasopharyngeal thermometer for body temperature control and a three-dimensional transducer for intraoperative transesophageal echocardiography were used.

After positioning the patient, markings were made on the thorax surface for trocar introduction. After systemic heparinization, peripheral cannulation of the femoral vein and artery, as well of the right internal jugular vein were performed under trans-esophageal echocardiography guidance. Prior to trocar introduction, the right lung was isolated from ventilation. The trocar for the micro camera was positioned prior to its introduction. The right hemithorax was inspected and then the remaining trocars were introduced. The DaVinci ${ }^{\circledR}$ robotic system (Intuitive Surgical, Inc., Sunnyvale, CA, USA) was docked.

Through the second right intercostal space (RICS) in the mid-axillary line (MAL), the Chitwood transthoracic clamp was introduced (Fehling Instruments GMBH \& CO. KG, Karlstein, Germany). Cardiopulmonary bypass (CPB) was established and the patient was cooled to $28^{\circ} \mathrm{C}$. With the aid of videothoracoscopy, the ascending aorta was clamped and punctured with a $30-\mathrm{cm}$ metal needle (Geister Medizintechnik, Tuttlingen, Germany) for the administration of antegrade crystalloid cold cardioplegic solution Custodiol HTK (Köhler Chemie GmbH,
Bensheim, Germany). With the aim of reducing the effects of air embolism, carbon dioxide was insufflated from the time of left atrial opening to the time of closure.

The mitral valve was inspected prior to the procedure and then repaired (Figure 2) or replaced. Under transesophageal echocardiographic monitoring, the heart was thoroughly de-aired, and CPB was discontinued. The femoral and jugular cannulas were removed, and heparin was reverted using protamine at a 1:1 ratio. The right hemithorax was drained and the drain was exteriorized through the trocar port of the right robotic arm, directed to the interior of the pericardial sac.

Two patients had atrial fibrillation associated with mitral valve disease, and underwent mitral valve repair. The first patient underwent epicardial ablation with electrical isolation of the pulmonary veins using the Cobra Adhere XL (Estech, San Ramon, CA, USA) system, as described by Bevilacqua et al. (9), prior to the establishment of CPB. The second patient underwent endocardial ablation of the left atrium with Cardioblate ${ }^{\circledR}$ Surgical Ablation Monopolar System Medtronic, Inc. (Minneapolis, MN, USA) (10).

\section{Correction of atrial septal defect}

Surgical access for ASD repair was similar to the approach used in robotic-assisted mitral valve surgery (11). In ASD repair, the right atrial chamber was accessed after isolation of the superior and inferior venae cavae. The introduction of the micro camera into the right atrium enabled the ASD to be visualized and corrected using a bovine pericardial patch (12). In all seven cases of this series, ASDs were classified as ostium secundum.

\section{Intracardiac tumor resection}

Robotic-assisted resection of intra-cardiac tumors also used a similar surgical approach to that described for roboticassisted mitral valve surgery (13).

For tumours located in the left atrium, left atriotomy 
was performed in the region of the right pulmonary veins following cardioplegia. In one patient, the tumor was attached to the left posterior atrial wall, which was broadly resected and replaced by a bovine pericardial patch. In a second patient, the tumor was adherent to the atrioventricular septum, anterior to the anterior cusp of the mitral valve. When the tumor was found in the right atrium, this chamber was opened and, without using cardioplegia, part of the right atrial free wall that the tumor adhered to was resected, and primary suture of the right atrium was performed.

\section{Pericardiectomy}

Robotic-assisted pericardial resection was performed by positioning ports in a similar way to that described for mitral valve repair. These procedures were performed offpump. The pericardium was broadly resected, parallel to the right phrenic nerve, up to the region corresponding to the anterior aspect of the left ventricle.

\section{Treatment of isolated atrial fibrillation}

In the treatment of isolated AF, patient preparation, positioning and markings were the same as described for robotic mitral valve surgery. Prior to the introduction of trocars, the right lung was isolated. The micro camera was introduced into the fourth RICS, in the anterior axillary line (AAL). The right thorax was inspected and the remaining trocars were introduced. The $\mathrm{DaVinc}{ }^{\circledR}$ robotic system (Intuitive Surgical, Inc., Sunnyvale, CA, USA) was docked.

After opening the pericardial sac, the superior and inferior venae cavae were dissected and a guide was introduced through the transverse sinus, posterior to the left atrium, directly below the pulmonary veins, until emerging in the oblique sinus. The ablation probe Cobra Adhere XL (Estech, San Ramon, CA, USA) was introduced using a guide, and was positioned for ablation. After correctly positioning via endoscopic approach, the epicardial ablation system was activated. After the first ablation, the ablation line was visually checked and, later, electrically checked using CARTO 3DTM (Biosense Webster, Johnson \& Johnson, Diamond Bar, California, USA) device for electroanatomical mapping. If a gap was identified by the electroanatomical system, it was corrected by the electrophysiologist.

\section{Totally endoscopic coronary artery bypass}

A technique described by Bonatti (14) was used for totally endoscopic coronary artery bypass (TECAB). TECAB was performed off-pump via a left thoracic approach. After single-lung ventilation had been established with isolation of the left lung, a $12-\mathrm{mm}$ trocar was introduced in the fifth left ICS (LICS), for the introduction of the micro camera. Two trocars for the $8-\mathrm{mm}$ robotic instruments were inserted in the third and seventh LICS in the left AAL. Carbon dioxide was constantly insufflated in the left thorax thus ensuring room for dissection of the left internal thoracic artery (LITA).

After robotic dissection of the LITA, a robotic coronary stabilizer was used, which was introduced in the left hemithorax and connected to the fourth robotic arm. The left anterior descending (LAD) artery segment to be anastomosed was mechanically immobilized and occluded proximally and distally using a tourniquet. After completion of the anastomosis, a TTFM (Medstim, Oslo, Norway) flow-meter was used to check adequate flow through the anastomosis. No patients underwent hybrid approach with additional stenting to other vessels.

\section{Statistical analysis}

Continuous variables were expressed as mean, standard deviation (SD), median and range, and categorical data were expressed as count and percentage. The KaplanMeier method was used for survival analysis. Data analysis was performed using SPSS software (version 18.0; SPSS, Chicago, IL, USA).

\section{Results}

The preoperative diagnoses are demonstrated in Figure 3, and the procedures performed are summarized in Figure 4.

Twenty-seven patients $(69.2 \%)$ were male. The mean age was $51.3 \pm 17.9$ years, ranging from 20 to 83 years and the mean ejection fraction was $62 \pm 5 \%$. The New York Heart Association (NYHA) functional class I/II was observed in $69.3 \%(27 / 39)$. The most commonly documented comorbidities included systemic arterial hypertension (43.6\%), previous acute myocardial infarction $(10.3 \%)$ and diabetes $(7.7 \%)$. A history of cigarette smoking was recorded in $15.4 \%$ of patients.

The most common procedure performed was mitral valve surgery $(\mathrm{N}=20)$. In echocardiographic assessment, 18 patients showed severe mitral valve regurgitation (MR) due to degenerative change and two had double lesion due to rheumatic disease. Repairs were possible in 15 cases and 


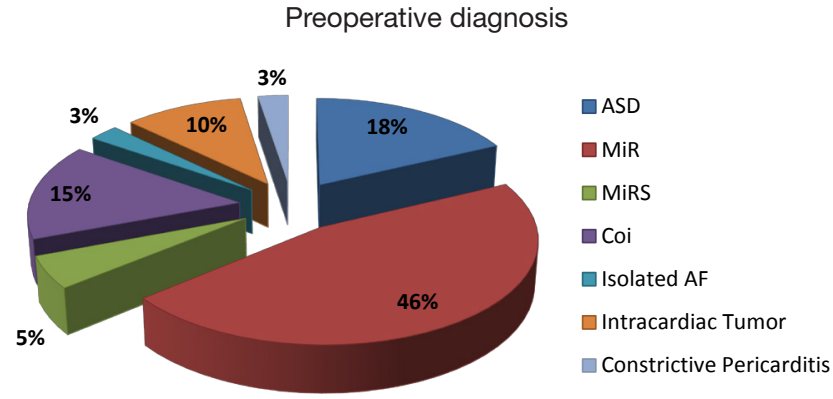

Figure 3 Preoperative diagnosis. ASD, atrial septal defect; MiR, mitral regurgitation; MiRS, mitral regurgitation and stenosis; Coi, coronary insufficiency; AF, atrial fibrillation.

Types of surgical procedures

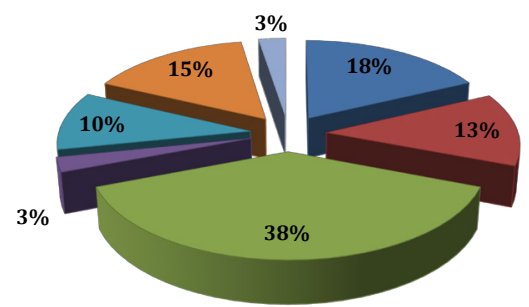

$\square$ Atrial Septoplasty $\square$ Mitral Valve Replacement $\square$ Mitral Valve Repair - Hybrid AFT

$\square$ Tumor Resection Coronary Artery Bypass Pericardiectomy

Figure 4 Types of surgical procedures. AFT, atrial fibrillation therapy.

five replacements were performed. Patent foramen ovale was identified in four patients in association with mitral valve disease, and atrial septal suture was performed. Two patients with mitral valve disease had atrial fibrillation and underwent mitral valve repair in addition to surgical ablation.

The remaining cases were assorted as follows: seven patients with ASD (17.9\%) were submitted to atrial septoplasty with bovine pericardial patch; six patients (15.3\%) underwent TECAB to the LAD due to severe stenosis $(>70 \%)$ and four had already undergone previous angioplasty and progressed with intracoronary stent stenosis. In all cases, the left internal thoracic artery (LITA) graft was used to revascularize the left anterior descending artery. Four (10.2\%) patients underwent tumor resection (three left atrial tumors and one right atrial tumor); one (2.5\%) underwent hybrid treatment of atrial fibrillation with epicardial-isolation of the pulmonary veins and endocardial ablation; and one patient underwent pericardiectomy.

All procedures are summarized in Table 1.

There were no intra-operative complications. The mean cardiopulmonary bypass and the mean aortic cross-clamp time are presented in Table 2, categorized by the type of procedure. TECAB, pericardiectomy and treatment of isolated atrial fibrillation were performed without the use of cardiopulmonary bypass.

After the procedure, all patients underwent intraoperative TEE showing successful valvuloplasty with absent or trivial mitral regurgitation, prostheses well positioned and functioning normally, no residual shunt after ASD closure and complete tumor resection.

Of the 39 patients, $32(82,0 \%)$ were extubated in the operating room immediately after surgery, and the remaining seven patients were extubated in an ICU with a median tracheal intubation time of 9 hours (ranging from 5.8 to 22 hours). The median ICU length of stay was 1 day (ranging from 0 to 25) and the median hospital length of stay was 5 days (ranging from 3 to 25). Endpoints are shown in Table 3, categorized by type of procedure.

All procedures were performed as planned, with none requiring conversion to sternotomy. Post-operative complications included one case of unilateral pulmonary edema (UPE) (2.5\%), two cases of atrial fibrillation with rapid ventricular response $(5.1 \%)$ and three re-explorations for bleeding (7.6\%). One patient developed a systemic inflammatory response syndrome (SIRS). Stroke was observed in one patient $(2.5 \%)$ on the $20^{\text {th }}$ postoperative day, two weeks after hospital discharge. No infections of the surgical sites were reported. One case of femoral pseudoaneurysm was reported following use of peripheral cannulation. However, this thrombosed spontaneously, with preserved arterial perfusion of the limb with no further complications.

In this series, two deaths (5\%) were reported in the early post-operative period. One patient had acute respiratory failure due to a bronchopneumonia on the $25^{\text {th }}$ postoperative day. A second patient died from complications of an ischemic stroke after hospital discharge on the $24^{\text {th }}$ postoperative day.

There were no late deaths and all patients are being regularly evaluated on an outpatient basis. Mean followup time was $1,126.33 \pm 734.14$ days, ranging from 24 to 2,118 days (Figure 5).

Transthoracic echocardiography (TTE) was obtained every 6 months in all non-TECAB patients. In those patients who underwent mitral valve repair, no significant mitral regurgitation was documented. At mid-term, there was no need for re-operation.

On the qualitative analysis, all patients showed a marked 


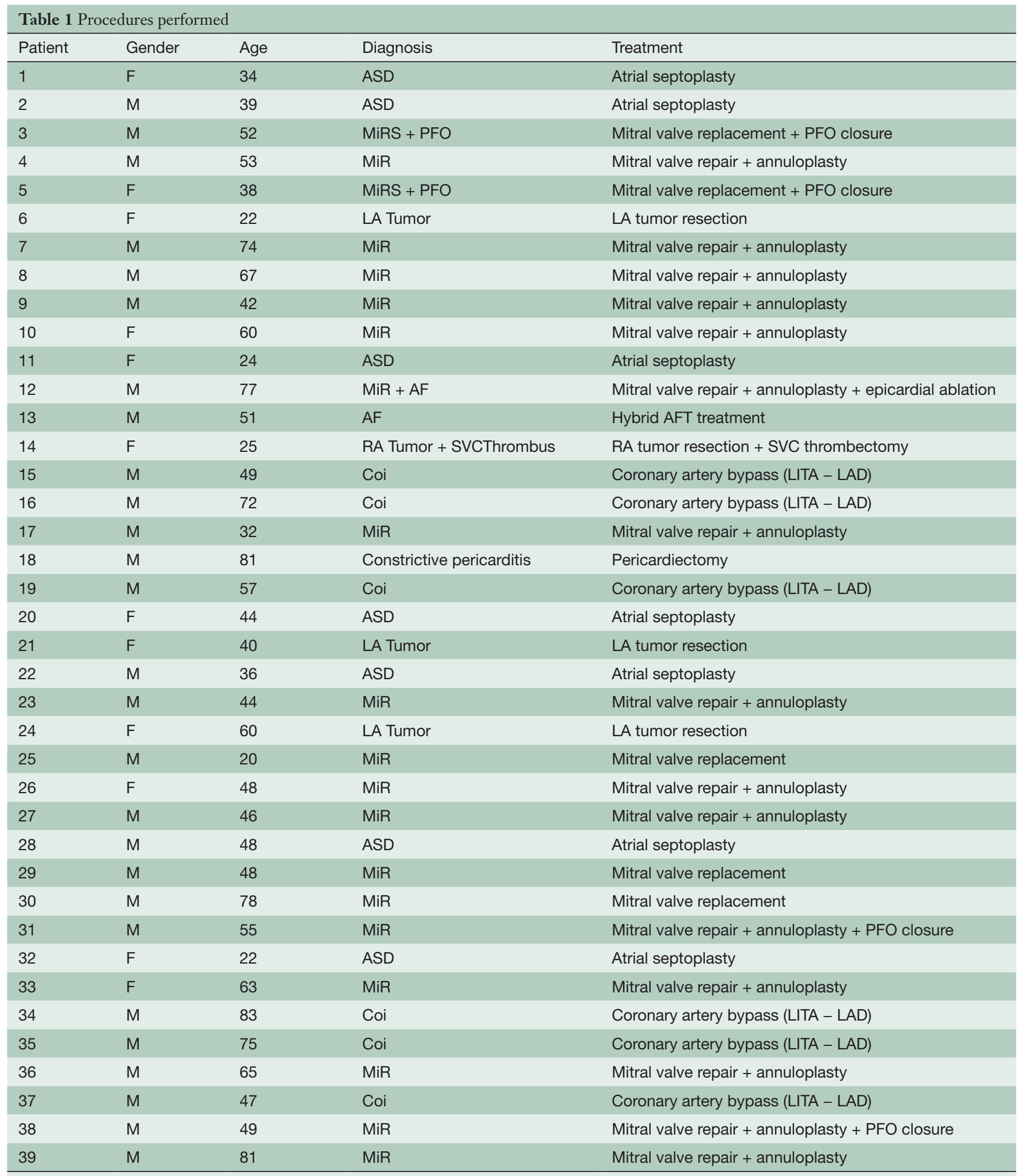

F, female; ASD, atrial septal defect; M, male; MiR, mitral regurgitation; MiRS, mitral regurgitation and stenosis; PFO, patent foramen ovale; LA, left atrium; AF, atrial fibrillation; RA, right atrium; SVC, superior vena cava; Coi, coronary insufficiency; LITA, left internal thoracic artery; $L A D$, left anterior descending artery. 
Table 2 Intraoperative data

\begin{tabular}{|c|c|c|c|c|}
\hline Type of procedure & \multicolumn{2}{|c|}{ Cardiopulmonary bypass time (min) } & \multicolumn{2}{|c|}{ Aortic cross-clamp time (min) } \\
\hline Atrial septoplasty & $160.06 \pm 86.10$ & $140-240$ & $117.18 \pm 71.71$ & $75-150$ \\
\hline Mitral valve repair & $151.72 \pm 97.00$ & $120-340$ & $112.36 \pm 78.43$ & $90-260$ \\
\hline Mitral valve replacement & $156.14 \pm 90.65$ & $170-280$ & $113.57 \pm 75.63$ & $120-210$ \\
\hline All & $154.92 \pm 94.22$ & $120-340$ & $114.48 \pm 75.66$ & $75-260$ \\
\hline
\end{tabular}

Table 3 Length of stay

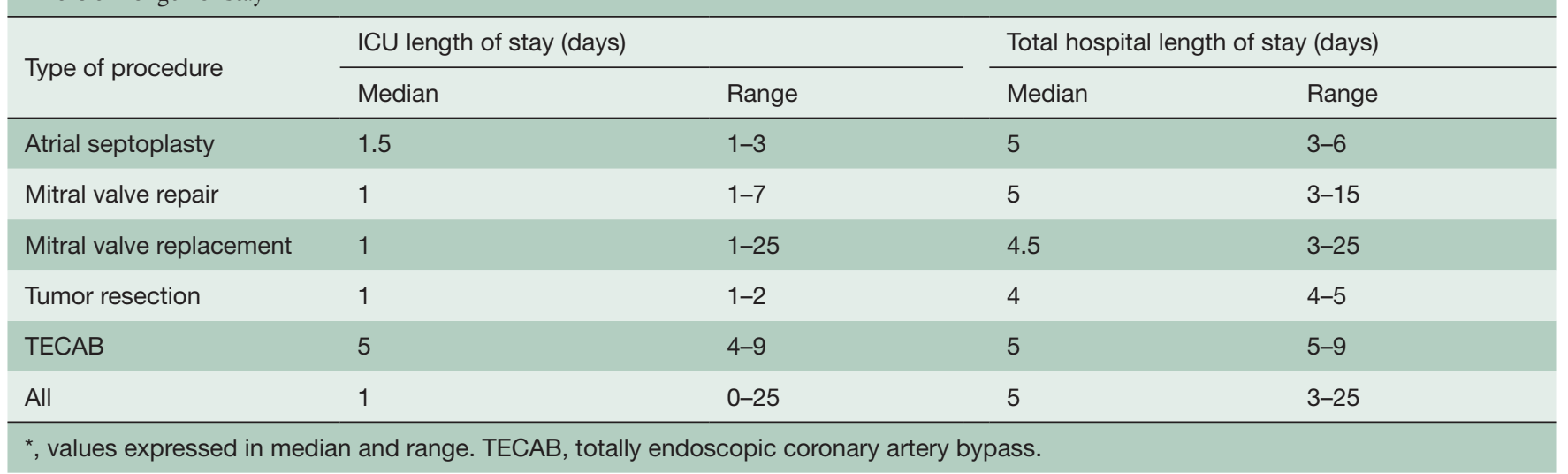

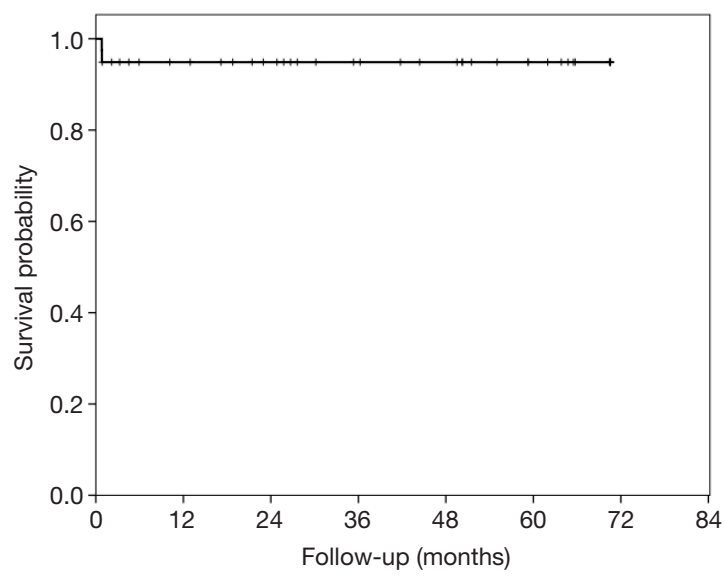

Figure 5 Kaplan-Meier survival curve. improvement in cardiovascular function, with NYHA functional class at pre-operative (I, 25.8\%; II, 43.5\%; III, $30.7 \%$ ) and post-operative (I, 84.6\%; II, 15.4\%; III, 0\%) intervals are shown in Figure 6.

\section{Discussion}

The use of minimally invasive cardiac surgery via minithoracotomy was, for many years, limited by the need for direct visualization of the heart and by the use of inappropriate instruments. With the incorporation of video-assisted techniques, it has been shown to be safe and effective in different surgical situations (15-19). However, the acquisition of new abilities such as manipulation 


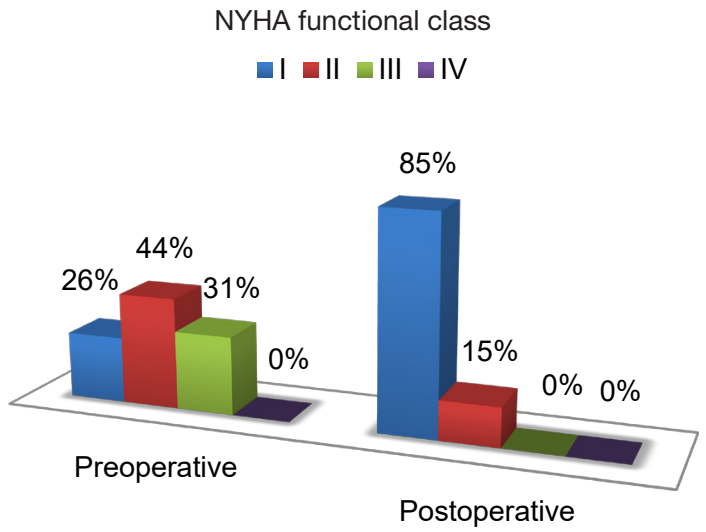

Figure 6 New York Heart Association functional class.

of longer instruments, the restricted operative field, replacement of direct vision by a two dimensional image and the change in tactile feedback represent a challenge to the surgeons. During the last decade, a variety of minimal-access approaches have been developed to access the mitral valve, from limited sternotomies to minithoracotomy assisted by videothoracoscopy. All had the same goals: to decrease surgical trauma and surgical complications leading to better patient recovery (20). The introduction of the $\mathrm{DaVinci}^{\circledR}$ robotic system significantly improved what can be visualized in the operative field, providing a three-dimensional image with ten times magnification. The use of the DaVinci ${ }^{\circledR}$ robotic system allows for better precision whilst performing cardiac surgeries through smaller incisions.

The most commonly performed procedure in this series was robotic mitral valve surgery. Mitral valve repair was possible in 15 patients, with a variety of techniques used: leaflet resections and reconstructions, chordal procedures (including chordal transfers and polytetrafluorethylene neochord constructions). Ring annuloplasty was performed in every case. The DaVinci robotic system represents a truly endoscopic approach to perform mitral valve surgery. The instrument tips are telemanipulated through seven degrees of ergonomic freedom that enable surgeons to perform complex mitral valve repairs through port incisions, providing access to the entire subvalvular chordal apparatus, papillary muscles and all leaflet topography (8). However, mitral valve repair is not always possible, particularly in severely calcified valves of rheumatic origin. In this series, five mitral valves were considered irreparable and replacement was performed. The benefits of robotic mitral valve replacement include smaller thoracotomy, better visualization and exposure of the valve, and easier suture handling in difficult anatomy (11).

The second most commonly performed procedure in this series was atrioseptoplasty. Patients had a shorter length of hospital stay and no documented complications, which is in accordance with the literature (21). The less common procedures in this series also benefited from robotic assistance. The robotic technique for coronary artery bypass surgery demonstrated reduction in surgical trauma whilst maintaining thoracic integrity. Many studies show reduction of the early postoperative time period with a faster return to full activity (14). Robotic technique for intracardiac tumors allowed for complete resection of the mass without sternotomy. Hybrid ablation of atrial fibrillation was facilitated by the robotic equipment, with successful maintenance of sinus rhythm in a period of 26 months of follow-up. Whilst most procedures discussed are suitable for robotic approaches, pericardiectomy is only possible when no significant calcification of the pericardium is present, as robotic instruments are delicate and sectioning thicker tissues may be difficult. In this series, pericardiectomy was easily performed for selected patients as no significant calcification was present in any case.

The major complications documented in this series were related to bleeding and arrhythmias. In three cases, bleeding with need for re-exploration occurred. In these cases, the original surgical access ports were used to perform surgical hemostasis, which was promptly achieved in all cases. In two cases, the source of bleeding was identified as being the intercostal vein. As a result of this finding, careful examination of all port incisions for haemostasis at the final revision is recommended. Atrial fibrillation with rapid ventricular response was documented in two cases; however both were successfully chemically reverted with intravenous amiodarone. Recent studies have documented the rate of post-operative atrial fibrillation to range from $5 \%$ to $40 \%$ in TECAB, and from $37 \%$ to $50 \%$ in heart valve surgery (22).

When comparing operative times, robotic cardiac surgery had prolonged times when compared with sternotomy, perhaps reflecting the complexity and learning curve associated with this new technology. Charland and colleagues (23) established that this learning curve can be overcome with adequate planning, gain of surgical volume and experience, with both approaches eventually comparable in duration (24). In this series, the greater $\mathrm{CPB}$ and cross-clamp times were not related to increased morbidity or complications. In fact, this series documented shorter length of ICU stay as well as total hospital stay. Furthermore, the majority of patients were extubated in 
the operating room. A downward trend in operative times in the last ten cases was observed as the surgeon evolved on the learning curve.

Despite advancements in cardiac surgery over recent decades, very little has been done to improve minimally invasive cardiac surgery (16). Surgical techniques are in constant change and no delay to follow this progress should be tolerated. However, the expansion of robotic cardiac surgery in Brazil and in other developing countries depends on public and private initiatives to create a health system guided by innovation and driven by quality.

There are important limitations to this study, primarily relating to its small, non-randomized, single-center cohort. Further studies involving larger randomized cohorts and long-term follow up should be considered to assess this promising and evolving technology.

In summary, this study supports the role of robotic cardiac surgery as a feasible, safe and effective alternative to sternotomy for the surgical correction of various intraand extra-cardiac pathologies. Adopting the robotic system has been a challenge in Brazil, where the lack of specific training and high initial costs of technology are the main impediments for a wide adoption. Nevertheless, overcoming these obstacles is essential to ensure that robotic cardiac surgery, as a commonplace in Brazil, may soon become a reality.

\section{Acknowledgements}

The authors would like to thank Dr. Wiley Nifong, Dr. Randolph Chitwood, Dr. Husam Balkhy and Mrs. Susan Bewick the ones who shared the knowledge and made robotic cardiac surgery become a reality in Brazil.

\section{Footnote}

Conflicts of Interest: The authors have no conflicts of interest to declare.

\section{References}

1. Lee JD, Srivastava M, Bonatti J. History and current status of robotic totally endoscopic coronary artery bypass. Circ J 2012;76:2058-65.

2. Chitwood WR Jr. Robotic cardiac surgery by 2031. Tex Heart Inst J 2011;38:691-3.

3. Bonaros N, Schachner T, Lehr E, et al. Five hundred cases of robotic totally endoscopic coronary artery bypass grafting: predictors of success and safety. Ann Thorac Surg 2013;95:803-12.

4. Suri RM, Antiel RM, Burkhart HM, et al. Quality of life after early mitral valve repair using conventional and robotic approaches. Ann Thorac Surg 2012;93:761-9.

5. Wikipedia. Minimally invasive procedures. Available online: https://en.wikipedia.org/wiki/Minimally_invasive_ procedures

6. Loeb JM, O'Leary DS. From the Joint Commission on Accreditation of Healthcare Organizations. JAMA 1995;273:1405.

7. Bonow RO, Carabello B, de Leon Jr. AC, et al. ACC/ AHA guidelines for the management of patients with valvular heart disease: A report of the American College of Cardiology/American Heart Association Task Force on practice guidelines (Committee on management of patients with valvular heart disease) 12 . J Am Coll Cardiol 1998;32:1486-582.

8. Chitwood WR Jr, Rodriguez E, Chu MW, et al. Robotic mitral valve repairs in 300 patients: a single-center experience. J Thorac Cardiovasc Surg 2008;136:436-41.

9. Bevilacqua S, Gasbarri T, Cerillo AG, et al. A new vacuumassisted probe for minimally invasive radiofrequency ablation. Ann Thorac Surg 2009;88:1317-21.

10. Sueda T, Nagata H, Shikata H, et al. Simple left atrial procedure for chronic atrial fibrillation associated with mitral valve disease. Ann Thorac Surg 1996;62:1796-800.

11. Gao C, Yang M, Xiao C, et al. Robotically assisted mitral valve replacement. J Thorac Cardiovasc Surg 2012;143:S64-7.

12. Poffo R, Celullare AL, Pope RB, et al. Robotic assisted minimally invasive surgery for atrial septal defect correction. Rev Bras Cir Cardiovasc 2012;27:488-90.

13. Gao C, Yang M, Wang G, et al. Excision of atrial myxoma using robotic technology. J Thorac Cardiovasc Surg 2010;139:1282-5.

14. Bonatti J, Schachner T, Bonaros N, et al. Robotically assisted totally endoscopic coronary bypass surgery. Circulation 2011;124:236-44.

15. Poffo R, Pope RB, Selbach RA, et al. Video-assisted cardiac surgery: results from a pioneer project in Brazil. Rev Bras Cir Cardiovasc 2009;24:318-26.

16. Poffo R, Pope RB, Toschi AP. Minimally invasive videoassisted atrial septal defect correction and myocardial revascularization. Rev Bras Cir Cardiovasc 2009;24:586-9.

17. Gao C, Yang M, Wang G, et al. Totally endoscopic robotic ventricular septal defect repair in the adult. J Thorac Cardiovasc Surg 2012;144:1404-7. 
18. Suri RM, Burkhart HM, Rehfeldt KH, et al. Robotic mitral valve repair for all categories of leaflet prolapse: improving patient appeal and advancing standard of care. Mayo Clin Proc 2011;86:838-44.

19. Bush B, Nifong LW, Chitwood WR Jr. Robotics in cardiac surgery: past, present, and future. Rambam Maimonides Med J 2013;4:e0017.

20. Poffo R. Cirurgia cardíaca minimamente invasiva. einstein. Educ Contin Saúde 2009;7:206-10.

21. Xiao C, Gao C, Yang M, et al. Totally robotic atrial septal defect closure: 7-year single-institution experience and follow-up. Interact Cardiovasc Thorac Surg

Cite this article as: Poffo R, Toschi AP, Pope RB, Montanhesi PK, Santos RS, Teruya A, Hatanaka DM, Rusca GF, Fischer $\mathrm{CH}$, Vieira MC, Makdisse MR. Robotic cardiac surgery in Brazil. Ann Cardiothorac Surg 2017;6(1):17-26. doi: 10.21037/ acs.2017.01.01
2014;19:933-7.

22. Mostafa A, El-Haddad MA, Shenoy M, et al. Atrial fibrillation post cardiac bypass surgery. Avicenna J Med 2012;2:65-70.

23. Charland PJ, Robbins T, Rodriguez E, et al. Learning curve analysis of mitral valve repair using telemanipulative technology. J Thorac Cardiovasc Surg 2011;142:404-10.

24. Suri RM, Taggarse A, Burkhart HM, et al. Robotic Mitral Valve Repair for Simple and Complex Degenerative Disease: Midterm Clinical and Echocardiographic Quality Outcomes. Circulation 2015;132:1961-8. 